\title{
On the Modal Aspects of Causal Sets
}

\section{Tomasz Placek $^{1}$ (D)}

Received: 22 January 2019 / Accepted: 17 June 2019 / Published online: 29 June 2019 (c) The Author(s) 2019

\begin{abstract}
The possibility question concerns the status of possibilities: do they form an irreducible category of the external reality, or are they merely features of our cognitive framework? If fundamental physics is ever to shed light on this issue, it must be done by some future theory that unifies insights of general relativity and quantum mechanics. The paper investigates one programme of this kind, namely the causal sets programme, as it apparently considers alternative developments of a given system. To evaluate this claim, we prove some algebraic facts about the sequential growth of causal sets. These facts tell against alternative developments, given that causal sets are understood as particular events. We thus interpret causal sets as multi-realisable objects, like states. This interpretation, however, is undermined by an argument for the probabilistic constraint of general covariance, as it says that multiple paths along which a causal set is produced are not physically different.
\end{abstract}

\section{Introduction: The Possibility Question}

In "Dilemma of determinism" (1884) William James succinctly contrasts the two camps in the the modality debate. ${ }^{1}$ One camp admits that "possibilities may be in excess of actualities", [...] [ so that ] actualities seem to float in a wider sea of possibilities from out of which they are chosen". The other camp, which he called "determinists", says that possibilities "exist nowhere, and that necessity on the one hand and impossibility on the other are the sole categories of the real. Possibilities that fail to get realized are [...] pure illusions: they never were possibilities at all.[...] The cloud of alternatives our minds escort this mass of actuality withal is a cloud of sheer deceptions, to which 'impossibilities' is the only name that rightfully belongs".

\footnotetext{
1 The support of the WSN2017 programme of the Polish Foundation for Science is gratefully acknowledged. I am indebted to David Rideout, David Meyer, and the participants of their seminar for discussions on causal sets. My special thanks go to Craig Callender.
}

$\bowtie$ Tomasz Placek

Tomasz.Placek@uj.edu.pl

1 Philosophy Department, Jagiellonian University, Kraków, Poland 
James made two comments which are particularly pertinent to the debate between the two camps. The first, and to which we full-heartedly subscribe, is that the issue is "a perfectly sharp one", or, as he says: "The truth must lie with one side or the other, and its lying with one side makes the other false". His second remark voiced scepticism as to whether science can resolve the issue. In his view, scientific conclusions are based on facts alone, but as for "the evidence of existing facts, the possibility-question must remain a mystery never to be cleared up." We take issue with this view, since (we think) it is based on an oversimplified view on the relations between scientific theories and experimental data. We tend to think of the possibility question as concerning a high-level claim, comparable (yet still more abstract) to the question of whether matter is continuous or corpuscular. Given that at the end of the nineteenth century theoretical and experimental data persuaded the scientific community to accept the corpuscular view, so we hope that future science might help to resolve the possibility question.

The second camp tends to be associated with Humean positions (although not necessarily with the views of the historical David Hume). However, the progress in the development of neo-Humean theories of laws of nature urges caution in this regard. Today's neo-Humeans countenance indeterminism, posing the question whether our world is deterministic or not. The neo-Humeans accomplished a rare feat of elucidating indeterminism in non-modal terms, in contrast to a large modal tradition. To highlight an otherwise well-known neo-Humean account of laws of nature, the MillRamsey-Lewis best-system account identifies laws of nature with the theorems of all true deductive systems that strike the best balence with respect to strength and simplicity. A system of laws is then said to be indeterministic if it is true about a pair of divergent worlds, i.e., qualitatively different worlds, whose initial segments are qualitatively alike. Finally, our world is called "indeterministic" if its set of laws comprises an indeterministic system of laws [7]. Note that the account appeals to concepts such as the linguistic description of a world, axiomatisation, strength and simplicity of an axiomatic system, the balance between the last two features, and does not invoke any modal notions. The resulting notions of laws and indeterminism depend on particularities of a language in which a world's description is stated, as well as on the understanding of strength, simplicity, and the balance between the two, the latter being rather properties of our conceptual framework than features of the objective non-linguistic and non-conceptual reality. Thus, neo-Humean indeterminism falls somewhat unlikely under "a category of the real". Furthermore, arguably neo-Humean indeterminism and modal indeterminism are not co-extensional concepts. The best system account may deliver the verdict "a world is indeterministic" that results from the laws of nature not being complete enough (since best balance sacrifies a certain degree of strength in this regard), whereas the world in question is flat, possibility-wise. In the opposite direction, a not complete enough system of laws might fail to discern a pair of diverging worlds, and thus gives the verdict of determinism, where possibility-wise indeterminism reigns.

There are two morals to be taken from this neo-Humean story. First, for clarity's sake, given that the rejection of possibilities can be combined with indeterminism, we will not phrase the possibility question as concerning determinism (in contrast to James). Second, while subscribing to James' succinct formulation, "are possibilities a category of the real", we add the gloss that the real is to be meant as the objective 
reality, independent of its linguistic characterisation, or its representation in this or that cognitive framework.

Given the high-brow formulation of the possibility question, how is fundamental science meant to help resolve it? To reflect first on the current situation, our best theories, namely quantum mechanics and general relativity, are not of much help. General relativity is the theory of the large, and is silent or problematic about small objects, like particles and their evolutions. Although it admits space-times that are somewhat reminiscent of alternative future possibilities, they always come with causal anomalies, which makes the qualification "future possibilities" problematic. ${ }^{2}$ Quantum mechanics, the theory of the small, initially appears to be more up to the task, as by a handbook description of its measurement algorithm, it yields the set of possible results of a particular measurement. But, to recall, the measurement algorithm is in conflict with the evolution law of quantum mechanics, Schrödinger's equation, which describes deterministic evolutions of quantum states. The conflict is known as the measurement problem of quantum mechanics, and its resolution is the aim of the interpretations of quantum mechanics. According to some interpretations, moreover, a measurement's possible results are understood as determined by values of a hidden factor, so the results are not alternative possibilities, in the required sense. In any case, without resolving the measurement problem, quantum mechanics can hardly shed light on the possibility question.

The present predicament suggests that we need to look at a theory of the large and the small, one which provides a unification of the quantum and general relativity. Currently there are a few programmes of that kind, known as programmes in quantum gravity. However, a question which presents itself is what features of a theory are relevant to the possibility question. How are possibilities represented in the mathematical structures of the theory? In short, what should we look for if we are interested in the question of possibility? We do not know what a general answer to the queries is, however intriguing they are. Therefore, we follow in this paper a simple instinct that links possibilities to the admittance of alternative evolutions of a theory's system, or (somewhat differently), to the existence of crossing trajectories in a theory's state space. From this perspective, the causal sets programme in quantum gravity initially seem quite promising. In the remainder of this paper we will investigate modal aspects of this programme.

The plan of the rest of this paper is as follows. In Sect. 2 we introduce the causal sets programme and then in Sect. 3 we discuss causal order- the prominent concept of this programme. Then Sect. 4 presents our mathematical results concerning sequential growth: it gives a set-theoretical reading of the theoretical physics style heuristic arguments of the programme and then exhibits the proofs of some facts about evolutions of causal sets. These facts are a formal skeleton of the paper, as they undermine the cogency of the notion of alternative possibilities in the model of sequential growth. Section 5 thus turns to formal metaphysics to discuss under what conditions events can happen together. The conclusion of this section is that causal sets should be interpreted as repeatable states rather than complex non-repeatable events. Next Sect. 6 discusses this interpretation, focusing in particular on its probabilistic aspects. It is

\footnotetext{
2 Technically, these are the so-called non-isometric extensions of a maximal globally hyperbolic space-time, cf. Chruściel and Isenberg [4].
} 
argued there that one of the assumed probabilistic rules, called general covariance, implies the rejection of physical reality of alternative possible paths leading to a given state. Finally, Sect. 7 discusses some options of how to preserve the concept of alternative possibilities in the theory of sequential growth. We begin with introducing causal sets programme.

\section{On the Causal Sets Programme}

Our concern in this paper is indeterministic (modal) aspects of the causal sets programme, which was launched by Raphael Sorkin and collaborators. ${ }^{3}$ This is a programme in quantum gravity that is based on two ideas: (i) a deep structure of quantum space-time, which the theory identifies with a causal set ("causet", in short), is discrete, and (ii) a causet is partially ordered by a causally interpreted ordering. The programme advances a few further claims that might as well draw a metaphysician's attention. To quote,

Another [feature of the theory] is a complete departure from determinism even at the fundamental scale, when all aspects of the "state of the system" are "known", the classical limit of the theory is postulated to be stochastic in nature. [...] Unitarity will likely have to be abandoned to formulate the quantum theory in a discrete setting. [...] Locality as a fundamental physical principle seems to be abandoned. [11, p. 109].

To add one more metaphysically controversial doctrine, there is real becoming according to the programme. That is, the universe is thought of as growing in consecutive stages, where the stages are defined in causal rather than temporal terms. Time and, generally, space-time, is constituted in the growth.

As expected, the sheer number of controversial claims has commanded attention of metaphysicians, philosophers of science, and physicists. The programme has been discussed, criticised, or defended from many perspectives. For a discussion from a philosophy of physics perspective, see Wüthrich and Callender [15], Wüthrich [14], and the references therein.

In what follows, I will focus on a somewhat less discussed feature of the programme: whether it represents possibilities as a separate category of physical reality. Recent decades have seen significant progress in the mathematically rigorous modelling of modalities, both in modal logics and formal metaphysics. Thus, the interaction between these philosophical traditions and the causal sets programme can be both illuminating and fruitful for both parties, I believe. From this perspective we will investigate the model of sequential growth that Rideout [11] and Rideout and Sorkin [12] put forward. Since the concept of ordering is crucial for modal issues, we focus on it in the next section.

\footnotetext{
3 Cf. Bombelli et al. [2]; for more info on the programme, see the causal sets reference webpage, www. phy.olemiss.edu/ luca/Topics/st/causal_sets.html.
} 


\section{Causal Order}

As a causet is a partial order $\preceq$, i.e., a reflexive, antisymmetric, and transitive order, ${ }^{4}$ that is further subject to a local finiteness assumption (see below), we focus first on the order. Calling the order "causal" indicates that it is to generalize the causal ordering of general relativity, GR, defined as

$x \preceq_{G R} y$ iff there exists a future-directed causal curve from $x$ to $y$.

The focus on this concept of general relativity comes from a diagnosis that "of all the major concepts in our current best theories, the spacetime causal order from general relativity and the path integral from quantum theory will survive the coming revolution" [5, p. 247], which in turn is based on a number of mathematical results, a particularly important one being Malament's [9] theorem. The theorem says that for an important class of GR space-times (the so-called future and past distinguishing spacetimes), a causal isomorphism preserves the metric structure up to a conformal factor. This is typically glossed by saying that causal ordering determines metric structure up to conformal factor. 5

Since a null-curve is causal, and a point can be connected to itself by a null-curve, $\preceq$ is reflexive. In some contexts, however, one may prefer to work with a related strict order (i.e., irreflexive and transitive) rather than a partial causal order $\preceq$. This strict order is defined by the equivalence $x \prec y \Leftrightarrow x \preceq y \wedge x \neq y$. Depending on the context, we will freely switch between $\preceq$ and $\prec$.

Irreflexivity and transitivity of $\prec$ imply asymmetry, $x \prec y \Rightarrow \neg(y \prec x)$. Asymmetry of $\prec$ as well as anti-symmetry of $\preceq$ in turn prohibit loops, $x \prec y \wedge y \prec x$ (or $x \preceq y \wedge y \preceq x \wedge x \neq y$ ). The prohibition of causal loops signals a departure from the general relativistic causal ordering, which permits such loops. This remark is not intended as criticism, since causal loops in GR are often seen as anomalous, or an artefact of the theory that allows for too many models, with many unphysical ones. ${ }^{6}$ A more worrisome observation is that causal ordering of GR seems to have deterministic underpinnings. After all, globally hyperbolic space-times have the initial value problem (IVP) well-posed, by the Choquet-Bruchat and Geroch theorem [3], meaning that there is a unique (up to isometry) maximal globally hyperbolic spacetime that extends an initial data set and satisfies Einstein's field equations. As globally hyperbolic space-times are often considered generic models of GR, the result provides evidence for the determinism of GR, if restricted to generic space-times. Thus, restricting ourselves to globally hyperbolic space-times, if we have $x \preceq_{G R} \quad y$ and $x \preceq_{G R} z$ and know that $x$ belongs to space-time $M$, then $y$ and $z$ must both belong to the same space-time $M$. This is to be contrasted with a modal-temporal ordering $<$, understood as "it might happen in the future of". For example, the fact that Toss $<$ Heads and Toss $<$ Tails does not entail that Tails and Heads can happen together

\footnotetext{
${ }^{4}$ Reflexive means that $x \preceq x$, anti-symmetry means that $x \preceq y$ and $y \preceq x$ imply $x=y$, and transitive means that $x \preceq y$ and $y \preceq z$ imply $x \preceq z$.

5 For a discussion of mathematical results underlying the causal sets program, see Reference Wüthrich [16].

6 By Censorship Conjecture, the generic space-times of general relativity are globally hyperbolic; in such space-times causal loops are absent. By equating "generic" with "physical" (a frequent move) one thus relegates GR causal loops to the realm of non-physical.
} 
(are co-possible). Here by Toss we understand a particular toss of a given coin, and Tails/Heads refer to alternative particular events of the coin landing tails up/heads up on a table. For indeterminism-friendly settings one might thus be tempted to venture into non-globally hyperbolic models, but they do not offer much help, either. Although in these cases there are non-unique space-times that extend a given initial data set, these space-times typically have causal anomalies such as causal loops, so GR causal ordering is then different from the asymmetric ordering postulated for causal sets.

The moral of these observations is that GR will not help us understand the causal sets ordering $\prec$ in an indeterminism-friendly way. As a result, the task of finding some structures in causal sets that can be interpreted as alternative possibilities becomes even more pressing.

The program gives prominence to the order concept that abstracts from individuality of what is ordered. The notion of set, with individuality of its elements, seems to be unnecessary and irrelevant given that order, volume, and quantum paths are sufficient for the deep structures of quantum space-times. Yet, in mathematics, one defines partial ordering as a partially ordered set, and causal sets theorists follow suit in their definitions. In their prose, however, they draw a distinction between "non-numbered" sets and "numbered sets". Further, diagrams depicting causal sets suggest that the only captured feature is the pattern of ordering (for non-numbered causets) or pattern of ordering plus a labelling function (for numbered causets). To produce corresponding representations, a natural move is to take recourse to equivalence classes (first case), with labelling added (second case). In the sections below, we provide such a representation and carry out the discussion of the causal sets in these terms. We focus on un-numbered causal sets, since by ignoring frame-dependent ordering they seem more fundamental.

Having rehearsed the basic notions of causal ordering, numbered causets, and nonnumbered causets, we next do some formal work. We give formal (set-theoretical) reading of theoretical physics style heuristic constructions of the causal sets programme. This is to exhibit mathematical meaning of these constructions, and, more importantly, to produce rigorous proofs of some facts about the process of sequential growth. The facts are important as they suggest that there is no room for alternative possible developments in the theory of sequential growth, contrary to what the programme promises.

\section{Facts About Un-numbered Causets}

A causet is a partially ordered set, $\langle C, \preceq\rangle$, with a local finiteness postulate,

$$
\forall x, z \in C \operatorname{card}\{y \in C \mid x \preceq y \preceq z\}<\infty
$$

i.e., the cardinality of elements lying between any two elements (that is, inside any Alexandrov's diamond) is finite. Recalling the terminology, a chain in $\langle C, \preceq\rangle$ is a linearly ordered subset of $C$, and a path between $x$ and $y$ in $C$ is a maximal chain between $x$ and $y$. 
To get a grip on paterns of order alone, in abstraction from any particular sets, we use slightly different definitions, and we do so in two steps. Yet, we begin with a set-relative notion:

Definition 1 (Proto-causets) Let $D$ be a countably infinite set. A proto-causet over $D$ is a non-empty partial order $\langle S, \preceq\rangle, S \subseteq D$, that satisfies Local Finiteness postulate (Eq. 1). The set of proto-causets over $D$ will be denoted by $\mathrm{PC}_{\mathrm{D}}$.

In accordance with the general idea that it is order rather than individual relata that is important, we next abstract from individuals (at least in part), focusing on appropriate equivalence classes. ${ }^{7}$

Definition 2 Let $\mathrm{OP}_{\mathrm{D}}$ be the set of order-preserving isomorphisms on $\mathrm{PC}_{\mathrm{D}}$. The set of causets over $D$ is: $\mathrm{C}_{\mathrm{D}}=\left\{[S, \preceq] \mid\langle S, \preceq\rangle \in \mathrm{PC}_{\mathrm{D}}\right\}$, where $[S, \preceq]=\left\{\left\langle S^{\prime}, \preceq^{\prime}\right\rangle \mid \exists R \in\right.$ $\left.\mathrm{OP}_{\mathrm{D}} R\left(\langle S, \preceq\rangle,\left\langle S^{\prime}, \preceq^{\prime}\right\rangle\right)\right\}$.

So causets are equivalence classes of proto-causets wrt order preserving isomorphisms.

We next introduce a relation of link on $\mathrm{PC}_{\mathrm{D}}$ that carries over to $\mathrm{C}_{\mathrm{D}}$, and which is to represent stages of the universe's growth.

Definition 3 (Links on $\mathrm{PC}_{\mathrm{D}}$ ) For $\langle S, \preceq\rangle,\left\langle S^{\prime}, \preceq^{\prime}\right\rangle \in \mathrm{PC}_{\mathrm{D}}$, we say that these two protocausets form a link in $\mathrm{PC}_{\mathrm{D}}$ iff there is $e \in S^{\prime} \backslash S$ s.t. $S^{\prime}=S \cup\{e\}, \preceq_{\mid S}^{\prime}=\preceq$, and $\forall x \in S^{\prime} e \npreceq^{\prime} x$. We say that $\langle S, \preceq\rangle$ is a parent, $\left\langle S^{\prime}, \preceq^{\prime}\right\rangle$ is a child, and $e$ is a new element in the link's child.

Note that by this definition a new element in a child cannot be below an element of the parent. Thus, the child's new element is placed either above a maximal chain in the parent, or space-like related (henceforth $S L R$ ) to all elements of the parent.

For the record, here is how the above definition carries over to causets:

Definition 4 (Links on $\mathrm{C}_{\mathrm{D}}$ ) For $A, B \in \mathrm{C}_{\mathrm{D}}$, we say that $\langle A, B\rangle$ is a link in $\mathrm{C}_{\mathrm{D}}$ iff there are $\langle S, \preceq\rangle$ and $\left\langle S^{\prime}, \preceq^{\prime}\right\rangle$ in $\mathrm{PC}_{\mathrm{D}}$ such that $A=[S, \preceq], B=\left[S^{\prime}, \preceq^{\prime}\right]$ and $\langle S, \preceq\rangle$ and $\left\langle S^{\prime}, \preceq^{\prime}\right\rangle$ form a link in $\mathrm{PC}_{\mathrm{D}}$.

For links in $C_{D}$ we use the same terminology of parent, child, and the child's new element as for the corresponding concepts in $\mathrm{PC}_{\mathrm{D}}$.

Let us observe that there are causets with single parents, and causets with multiple parents. As for the former, any causet based on a chain, or based on an anti-chain, has a single parent. The simplest two-parents causet is $\bullet \bullet$ (the parents are $\boldsymbol{\bullet}$ and $\bullet \bullet$ respectively). On the other hand, any two finite causets have a common ancestor, the singleton-based causet $\bullet$.

Since models of sequential growth use only finite causets (or, to be precise, causets based on finite proto-causets), from now on we restict our attention to the sets $\mathrm{PC}_{\mathrm{D}}^{\mathrm{fin}}$ and $\mathrm{C}_{\mathrm{D}}^{\mathrm{fin}}$ of finite proto-causets and causets based on finite proto-causets, respectively, where $D$ is countably infinite.

Links in $C_{D}^{\text {fin }}$ might serve the role of what in mathematical jargon is called covering relation, that is, the ordering that is no more breakable by transitivity into its constituents. In this spirit we use links to define an ordering on $\mathrm{C}_{\mathrm{D}}^{\mathrm{fin}}$ :

\footnotetext{
7 The relativisation to a base set $D$ still remains, but it can be removed at the cost of using somewhat more extricate mathematics.
} 
Fig. 1 Although $S_{A} \subsetneq S_{B}$, it is not the case that $A<B$, where $A=\left[S_{A}, \prec_{A}\right]$ and $B=\left[S_{B}, \prec_{B}\right]$, since the only way to add the new element to $S_{A}$ is to place it below an element of $S_{A}$
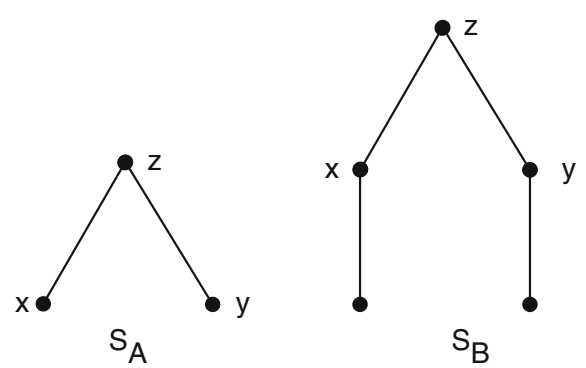

Definition 5 (Ordering of $\mathrm{C}_{\mathrm{D}}^{\mathrm{fin}}$ ) For $A, B \in \mathrm{C}_{\mathrm{D}}^{\mathrm{fin}}, A<B$ iff there is a finite sequence $\left[S_{1}, \preceq_{1}\right],\left[S_{2}, \preceq_{2}\right], \ldots\left[S_{n}, \preceq_{n}\right]$ in $C_{\mathrm{D}}^{\text {fin }}$ s.t. $A=\left[S_{1}, \preceq_{1}\right], B=\left[S_{n}, \preceq_{n}\right]$ and for every $i: 1 \leq i<n,\left[S_{i}, \preceq_{i}\right]$ and $\left[S_{i+1}, \preceq_{i+1}\right]$ form a link in $\mathrm{C}_{\mathrm{D}}^{\mathrm{fin}}$.

We subsequently show that $<$ is a strict ordering on $C_{D}^{\text {fin }}$ :

Fact $6<$ is a irrreflexive and transitive relation on $\mathrm{C}_{\mathrm{D}}^{\mathrm{fin}}$.

Proof $<$ is irrreflexive because no $\langle[S, \preceq],[S, \preceq]\rangle$ is a link in $\mathrm{C}_{\mathrm{D}}^{\mathrm{fin}}$. For transitivity of $<$, let the sequence $\left[S_{1}, \preceq_{1}\right],\left[S_{2}, \preceq_{2}\right], \ldots,\left[S_{n}, \preceq_{n}\right]$ be a witness for $A<B$, and the sequence $\left[S_{1}^{\prime}, \preceq_{1}^{\prime}\right],\left[S_{2}^{\prime}, \preceq_{2}^{\prime}\right], \ldots,\left[S_{m}^{\prime}, \preceq_{m}^{\prime}\right]$ be a witness for $B<C$. Since $B=\left[S_{n}, \preceq_{n}\right]=\left[S_{1}^{\prime}, \preceq_{1}^{\prime}\right]$, the concatenation of these two sequences (with $\left[S_{n}, \preceq_{n}\right]$ and $\left[S_{1}^{\prime}, \preceq_{1}^{\prime}\right]$ identified) is a witness for $A<C$.

As already remarked, irreflexivity and transitivity imply the asymmetry of $<$.

The question may present itself as to why should we opt to use the ordering of Definition 5 rather than the set-theoretical inclusion to order $C_{D}$. The answer is that the former is friendly to the concept of sequential growth in a way that the latter is not. Figure 1 show the two inclusion-related proto-causets none of which is an outgrowth of the other, as there is no sequence of parent-child links joining the two.

Another question is what are the consequences of working with equivalence classes of specified posets rather than specified posets themselves. What comes to the fore is that a parent in $\mathrm{PC}_{\mathrm{D}}^{\mathrm{fin}}$ has typically much more children than a parent in $C_{D}^{\mathrm{fin}}$. To calculate the number of children of a parent in $\mathrm{PC}_{\mathrm{D}}^{\mathrm{fin}}$ that has $m$ maximal elements, we count the number of ways a new element can be placed above these maxima, to obtain $\left(\begin{array}{c}m \\ 0\end{array}\right)+\left(\begin{array}{c}m \\ 1\end{array}\right)+\ldots+\left(\begin{array}{c}m \\ m-1\end{array}\right)+\left(\begin{array}{c}m \\ m\end{array}\right)$. That number is greatly reduced once we do equivalence classes of $C_{D}^{\text {fin }}$. Figure 2 shows a case of two children in $\mathrm{PC}_{\mathrm{D}}^{\mathrm{fin}}$ giving rise to one child in $\mathrm{C}_{\mathrm{D}}^{\mathrm{fin}} .8$

As we have just learned, $C_{D}^{\text {fin }}$ with the relation $<$ forms a strictly ordered set. We now investigate some further properties of it.

Fact 7 Any two finite causets $A, B \in \mathrm{C}_{\mathrm{D}}^{\mathrm{fin}}$ have a maximal lower bound $C$ in $\mathrm{C}_{\mathrm{D}}^{\mathrm{fin}}$, i.e., there is no $C^{\prime}>C$ that lower bounds $A$ and $B$. But some pairs of causets might have more than one maximal lower bound.

\footnotetext{
8 The figure suggests that the pasts of new elements in isomorphic children $\left\langle S_{B}, \prec_{B}\right\rangle$ and $\left\langle S_{D}, \prec_{D}\right\rangle$, i.e., $\{x, z\}$ and $\{y, z\}$, resp., are isomorphic as well, but generally this suggestion is wrong-for a counterexample, see Fig. 3.2 of Rideout [11].
} 
Fig. 2 Two proto-causets children of proto-causet $\left\langle S_{A}, \prec_{A}\right\rangle$. Since $\left\langle S_{B}, \prec_{B}\right\rangle$ and $\left\langle S_{D}, \prec_{D}\right\rangle$ are isomorphic, they yield just one child $\left[S_{A}, \prec_{A}\right]$ $\left\langle S_{B}, \prec_{B}\right\rangle$ and $\left\langle S_{D}, \prec_{D}\right\rangle$,

$\left[S_{A}, \prec_{A}\right]=\left[S_{B}, \prec_{B}\right]$ of causet
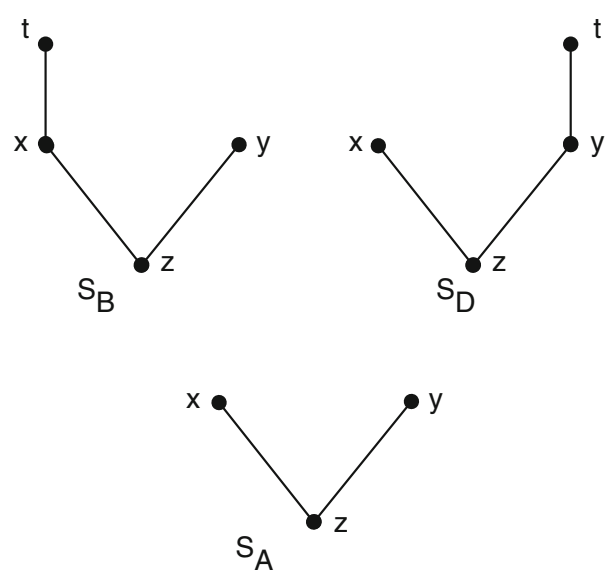

Fig. 3 Two causets $E$ and $F$ have two maximal lower bounds, $A$ and $B$. Two causets $A$ and $B$ have two minimal upper bounds, $E$ and $F$

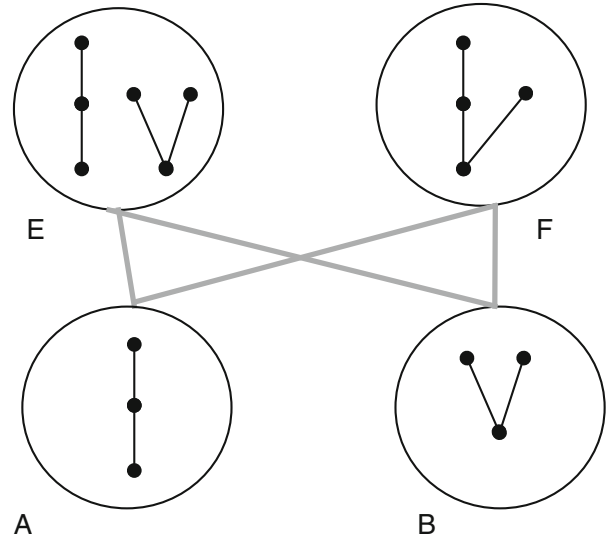

Proof If $A$ and $B$ are comparable by $<$ or identical, we are done. If $A$ and $B$ are incomparable by $<$, we pick a path $l$ leading from the one-element causet $\left[1, \preceq_{1}\right]$ to $A$ and a path $l^{\prime}$ leading from $\left[1, \preceq_{1}\right]$ to $B$. As these paths are finite and both start with the same element, there is a maximal element $m_{l l^{\prime}}$ in the paths' intersection. Since $A, B$ are finite, the number of paths from $\left[1, \preceq_{1}\right]$ to $A$ and from $\left[1, \preceq_{1}\right]$ to $B$ is finite, and hence the set of $m_{l l}$ 's is finite as well. This set thus has maximal elements, which by the constructions are maximal lower bounds of $A$ and $B$.

As for the part of the Fact starting with "But ...", consult Fig. 3 that depicts two finite causets with two maximal lower bounds. ${ }^{9}$

In the case of infinite causets, although any two are obviously lower bounded by $\left[1, \preceq_{1}\right]$, it is not known if they have maximal lower bounds.

Let us next turn to upper bounds:

Fact 8 Any two causets $A, B \in \mathrm{C}_{\mathrm{D}}^{\mathrm{fin}}$ have an upper bound $C$ in $\mathrm{C}_{\mathrm{D}}^{\mathrm{fin}}$. However, they might fail to have a least upper bound (supremum).

\footnotetext{
9 In the case of infinite causets, although any two are obviously lower bounded by $\left[1, \preceq_{1}\right]$, it is not known if they have maximal lower bounds. 
Proof Since $A$ and $B$ are based on finite proto-causets, while $D$ is countably infinite, we can pick disjoint subsets $S_{A}$ and $S_{B}$ of $D$ to represent $A$ and $B$, respectively. We then construct the so-called parallel composition of such two disjoint proto-causets $\left\langle S_{A}, \preceq_{A}\right.$ \rangle and $\left\langle S_{B}, \preceq_{B}\right\rangle$, then argue that the composition makes a proto-causet, and finally take the equivalence class that the proto-causet defines. The parallel composition of $\left\langle S_{A}, \preceq_{A}\right\rangle$ and $\left\langle S_{B}, \preceq_{B}\right\rangle$ is defined by putting $S=S_{A} \cup S_{B}$, and for $x, y \in S: x \preceq y$ iff $x \preceq_{A} y$ or $x \preceq_{B} y$. $\langle S, \preceq\rangle$ is a poset, and clearly $S \subseteq D$. Further, since $S$ is finite, being the sum of two finite sets, $\langle S, \preceq\rangle$ satisfies Local Finiteness postulate (Eq. 1). Accordingly, $\langle S, \preceq\rangle$ is a proto-cause and so it defines the equivalence class $C=[S, \preceq] \in \mathrm{C}_{\mathrm{D}}^{\mathrm{fin}}$.

It remains to be seen that $A<C$ and $B<C$. Given the finiteness assumptions, elements of $S_{B}=\left\{b_{1}, b_{2}, \ldots, b_{n}\right\}$ can be added consecutively to $S_{A}$ forming a finite chain of proto-causets:

$$
\left\langle S_{A}, \preceq_{A}\right\rangle,\left\langle S_{A} \cup\left\{b_{1}\right\}, \preceq_{1}\right\rangle,\left\langle S_{A} \cup\left\{b_{1}, b_{2}\right\}, \preceq_{2}\right\rangle, \ldots\left\langle S_{A} \cup\left\{b_{1}, b_{2}, \ldots b_{n}\right\}, \preceq\right\rangle,
$$

where each $\preceq_{i}$ is an appropriate restriction of $\preceq$, namely $\preceq_{i}=\preceq_{\mid S_{A} \cup\left\{b_{1}, b_{2}, \ldots, b_{i}\right\}}(i \leqslant n)$. Observe that in $S$ each element of $S_{A}$ is incomparable to each element of $S_{B}$, so indeed $\preceq_{i}$ is $=\preceq_{i+1}$ restricted to $S_{A} \cup\left\{b_{1}, b_{2}, \ldots, b_{i}\right\}$, i.e., $\preceq_{i}=\preceq_{i+1 \mid S_{A} \cup\left\{b_{1}, b_{2}, \ldots, b_{i}\right\}}$. Thus, any two consecutive elements of the above chain form a link, and the sequence of these links witnesses $A<C$. An analogous sequence of proto-causets leading from $S_{B}$ to $S$ shows that $B<C$ as well.

For the second part of the Fact (beginning with "However ..."), see Fig. 3: it exhibits two causets that have two minimal upper bounds. i.e., they have no least upper bound. ${ }^{10}$

An interesting consequence of the last two facts is that $\left\langle C_{D}^{f i n},<\right\rangle$ does not form a lattice, so it is not a Boolean algebra. That cast doubts on a motivation for causets, which was a construction of a Boolean algebra, so that the logic of causets-based quantum gravity be classical. Reference [13, p. 366] aims, for instance, to obtain a Boolean algebra by defining events as finite subsets of causets:

Let us write $A$ for the space of all events. Structurally, $A$ is a Boolean algebra, meaning that union, intersection, complementation and symmetric-difference are defined for it. In logical terms these correspond respectively to the connectives or, and, not, and xor.

One can of course take a Boolean algebra of subsets of a finite set of causets. This algebra, however, is distinct from partial order $\left\langle C_{D}^{f i n},<\right\rangle$, which is not even a lattice. In particular, the algebra is orderred by the set-theoretical inclusion $\subseteq$, not the causal ordering $<$. It is not at all clear how to wed the Boolean algebra idea to the basic structure $\left\langle\mathrm{C}_{\mathrm{D}}^{\mathrm{fin}},<\right\rangle$.

The last formal topic in this section are the maximal causets which are achievable in the process of sequential growth. We are invited to think of a child's new element as

10 Note, however, that numbered causets (which trace their ancestry) can be ordered into a tree-like branching structure, i.e., with a single minimal element and no two causets having an upper bound. For more on this, see the Appendix. 
a novel outgrowth of a parent, something that has become. We thus have finite chains of causets, i.e., related by $<$, representing finitely many stages of growth. So we can legitimately ask where the process of all the causets growing out of the singleton-based causet leads to. Since $\left\langle\mathrm{C}_{\mathrm{D}}^{\mathrm{fin}}, \leq\right\rangle$, with $A \leq B$ defined as $A<B$ or $A=B$, is a poset, by the Hausdorff maximality principle, we have this fact:

Fact 9 There are maximal chains in $\left\langle\mathrm{C}_{\mathrm{D}}^{\mathrm{fin}}, \leq\right\rangle$; any chain in $\left\langle\mathrm{C}_{\mathrm{D}}^{\mathrm{fin}}, \leq\right\rangle$ can be extended to a maximal chain; each maximal chain in $\left\langle\mathrm{C}_{\mathrm{D}}^{\mathrm{fin}}, \leq\right\rangle$ contains the singleton-based causet.

Moreover, we may identify a maximal outgrowth in the process of sequential growth with a union-like operation on maximal chains in $\left\langle C_{D}^{f i n}, \leq\right\rangle$, which exist by the Fact above. The identification is doable, as evidenced by this fact:

Fact 10 The union of the representatives of the elements of a maximal chain in $\left\langle\mathrm{C}_{\mathrm{D}}^{\mathrm{fin}}, \leq\right\rangle$ is a countably infinite proto-causet (so it yields as its equivalence class a countably infinite causet).

Proof Let $L=A_{1}, A_{2}, \ldots$ be a maximal chain in $\left\langle\mathrm{C}_{\mathrm{D}}^{\mathrm{fin}}, \leqslant\right\rangle$, guaranteed to exist by Fact 9 . Since $L$ is a maximal chain, every pair $A_{i}, A_{i+1}(i \in \mathbb{N})$ forms a link in $\mathrm{C}_{\mathrm{D}}^{\mathrm{fin}}$. There is thus a sequence $L^{\prime}$ of representatives of $A_{1}, A_{2}, \ldots$, namely $L^{\prime}=\left\langle S_{1}, \preceq_{1}\right\rangle$, $\left\langle S_{2}, \preceq_{2}\right\rangle, \ldots$ s.t. $A_{i}=\left[S_{i}, \preceq_{i}\right]$ and each pair $\left\langle S_{i}, \preceq_{i}\right\rangle,\left\langle S_{i+1}, \preceq_{i+1}\right\rangle$ forms a link in $\mathrm{PC}_{\mathrm{D}}^{\mathrm{fin}}$. Take now the union of the base sets of the representatives: $S^{*}=\bigcup\left\{S_{\gamma} \mid\left\langle S_{\gamma}, \preceq_{\gamma}\right.\right.$ \rangle$\in L^{\prime}$ \}. Clearly, $S^{*} \subseteq D$ as each $S_{\gamma} \subseteq D$. Since $S^{*}$ is the union of countably infinitely many finite sets, it is countably infinite. Define next a candidate for an ordering on $S^{*}$ : for $x, y \in S^{*}, x \preceq_{*} y$ iff there is $\left\langle S_{\gamma}, \preceq_{\gamma}\right\rangle \in L^{\prime}$ s.t. $x, y \in S_{\gamma}$ and $x \preceq_{\gamma} y$. The definition is consistent as it is impossible that for some $\left\langle S_{\gamma}, \preceq_{\gamma}\right\rangle,\left\langle S_{\eta}, \preceq_{\eta}\right\rangle \in L^{\prime}$ there are $x, y \in S_{\gamma} \cap S_{\eta}$ s.t. $x \preceq_{\gamma} y$ but $y \npreceq_{\eta} x$ (because the two are comparable by $<$ and the ordering on a parent is a restriction to the parent of the ordering on its child). For irreflexivity of $\preceq_{*}$, by the construction, for any $x \in S^{*}$ there is $\left\langle S_{\gamma}, \preceq_{\gamma}\right\rangle \in L^{\prime}$ and, as $x \npreceq_{\gamma} x$ for every $x \in S_{\gamma}, \preceq_{*}$ is irreflexive as well. For transitivity, let $\left\langle S_{\gamma}, \preceq_{\gamma}\right\rangle$ be a witness for $x<_{*} y$ and $\left\langle S_{\eta}, \preceq_{\eta}\right\rangle$ be a witness for $y \preceq_{*} z$. The two witnesses are comparable by $<$; let thus $\left\langle S_{\gamma}, \preceq_{\gamma}\right\rangle<\left\langle S_{\eta}, \preceq_{\eta}\right\rangle$ (the other case is exactly alike). Since this implies $S_{\gamma} \subseteq S_{\eta}$, it follows that $x, y, z \in S_{\eta}$. As $x<_{\gamma} y$, by consistency of $\preceq$-orderings in elements of $L^{\prime}, x<_{\eta} y$. This together with $y \preceq_{\eta} z$ implies, by transitivity of $\preceq_{\eta}$ and definition of $\preceq_{*}$, that $x \preceq_{*} z$. We thus established that $\left\langle S^{*}, \preceq_{*}\right\rangle$ is a poset, with $S^{*} \subseteq D$. It remains to see if it satisfies Local Finitely postulate as well. Note that for any $x, y \in S^{*}$, there is $S_{\gamma}$ s.t. $x, y \in S_{\gamma}$. By Local Finiteness for $\left\langle S_{\gamma}, \preceq_{\gamma}\right\rangle$, the set $\left\{z \in S_{\gamma} \mid x \preceq_{\gamma} z \preceq_{\gamma} y\right\}$ is finite. Also, if $z^{\prime} \in S_{\gamma}^{\prime} \backslash S_{\gamma}$ for $\left\langle S_{\gamma^{\prime}}, \preceq \gamma^{\prime}\right\rangle \in L^{\prime}$, then $z^{\prime} \npreceq \gamma^{\prime} y-$ by the definiton of links, since $y \in S_{\gamma}$. And by the definition of $\preceq_{*}$, if $x \preceq_{\gamma} z \preceq_{\gamma} \quad y$, then $x \preceq_{*} z \preceq_{*} y$. Combining these two observations, $\left\{z \in S_{\gamma} \mid x \preceq_{\gamma} z \preceq_{\gamma} y\right\}=\left\{z \in S^{*} \mid x \preceq_{*} z \preceq_{*} y\right\}$, which is finite. We thus have shown that $\left\langle S^{*}, \preceq_{*}\right\rangle \in \mathrm{PC}_{\mathrm{D}}$; hence it yields the equivalence class $\left[S^{*}, \preceq_{*}\right] \in \mathrm{C}_{\mathrm{D}}$.

We end this formal part by mentioning one more fact that has an interpretative significance. 
Fact 11 If a causet $A$ is based on a proto-causet $S_{A}$ containing two maximal chains of different length, then A has more than one parent.

Proof Since the proto-causet $S_{A}$ has two maximal chains of different length, it can be produced from two posets, each resulting from $S_{A}$ by shortening a different maximal chain of unequal length. (By shortening of a one-element chain we mean here the removal of this chain). Since these two posets differ wrt length of the two resulting chains, they are not equivalent, and hence define different causets, which by the constructions are parents of the original causet. And, as these parents are not identical, they are incomparable by $<$, so they belong to different maximal chains in $\left\langle\mathrm{C}_{\mathrm{D}}^{\mathrm{fin}},<\right\rangle$.

The consequence of this fact is that a typical causet has multiple parents, each parent belonging to a different maximal chain of causets, with all these chains containing the causet in question. ${ }^{11}$ This consequence creates tension with a modal interpretation of causets once we identify maximal chains of causets with possible histories producible in the process of sequential growth: after all, how can we have a parent from a different history than our own? Another problematic feature of causets is that any two causets have an upper bound, see Fact 8 . Before we discuss this tension further, we turn to formal metaphysics for help in elucidating the concept of alternative possibilities.

\section{Causets: Events or States?}

A natural interpretation of a causet's children is modal: children of a given parent are alternative possible stages of the universe's growth. Since a causet gives way to another causet, it does not contain the whole information about a maximal course of events that sequential growth can produce (an exception are causets produced by maximal chains in $C_{D}^{\text {fin }}$, as in Fact 10). A causet thus seems to be identifiable to what is sometimes called "partial history". This is to be contrasted with "history" simpliciter, that is, a maximal course of events. A partial history is compatible with more than one alternative history - otherwise it would not be partial. If one likes, one might think of histories simpliciter as causally-produced outgrowths of partial histories. In a similar vein, one may think of later causets (in the sense of $<$ ) as being more fine-grained (less partial) than their predecessors. While a partial history is compatible with more than one alternative fine-grainings of it, two alternative fine-grainings of a partial history cannot be compatible, and hence cannot be compatible with one and the same history. This is a familiar and intuitive tale, but, as we have already alluded, it seems to be in conflict with algebraic facts about causets.

The crux of the problem is the concept of alternative possibilities. How do we draw a distinction between alternative partial histories, and co-possible partial histories? The former cannot happen together, whereas the latter can, by being parts of a maximal course of events. An easier question is how to differentiate between alternative possible events and co-possible events. One might opt for a deflated responce that collapses copossibility with consistency, such as in "the set of events is co-possible iff a complete

11 The reference to typicality above reflects the relative rarity of causets in which all maximal chains have equal length. 
description of this set is consistent". Although this answer has some credibility among philosophers, it does not look like a cogent move in the context of fundamental physics, with its use of causal concepts, like $\preceq$ of sequential growth, which is a primitive causal notion, not reducible to logic. Now, an appeal to causal ordering, understood quite weakly, as “. . . can causally influence ...”, delivers a powerfull criterion of copossibility, due to Belnap [1]: if there is $z$ such that $x \preceq z$ and $y \preceq z$, then all three, $x, y$, and $z$ are co-possible. In plain words, if there is a possible later witness of $x$ and $y$, then $x$ and $y$ can really happen together; if the later witness actually happened, $x$ and $y$ happened as well. If in doubt whether a particular key stroke I might execute on my laptop, and a particular electromagnetic outburst on Andromeda that astrophysicists predict, can happen together, look for some third possible event, whose past comprises the two events in question. It is much easier to decide if the two events have happened together, as one faces an easier task of finding some actual event. Deciding whether the two events can happen together sets us a higher target of finding an appropriate possible event.

The later witness intuition is the implication from the existence of a later witness to a co-possibility. The other direction is more problematic, as a world might come to an abrupt end. The absence of a later witness of two events might signal a world's abrupt end rather than incompatibility of two events in question. ${ }^{12}$ Nevertheless, by having accepted the more problematic direction as well, we can use it to argue for negative verdicts. For instance, we observe that there is no possible event in the future of a particular coin toss that could be causally influenced by two possible results of it, landing Heads up and landing Tails up. Any possible event in the future of that flip can influenced by Heads up, or by Tails up, but not by both. Hence Tails up and Heads up are not co-possible.

The criterion of a later witness (with both directions) plays an essential role in Belnap's [1] Branching Space-Times (BST) theory. In mathematical parlance, a subset of a partially ordered set that satisfies the criterion is called an "upward directed subset". BST then defines histories as maximal upward directed subsets of a partially ordered set of all possible events.

Causets do not seem to satisfy the later witness intuition-unless the set $C_{D}^{\text {fin }}$ does not admit alternative finite causets. But if it does admit two alternative causets, then by Fact 8 there is a (finite) causet that upper bounds the two (wrt $<$ ). Recalling the reading of the order $<$ on $C_{D}^{\text {fin }}$, this means that starting from one alternative causet, or from another alternative causet, one arrives at the upper bound of the two by causal processes of sequential growth. One may thus wonder if the two causets were genuinely alternative to begin with. Somewhat similarly, causets do not seem to satisfy the demand that no event has a causal past containing incompatible parts; by Fact 11 a typical causet has many parents, with any two of them belonging to different maximal chains of causets.

The intuition of a later witness concerns events, so without an argument, we should not expect it to carry over to objects of other categories. Also, although elements of a causet are sometimes explicated as events (cf. Sorkin [13]), perhaps they are events

\footnotetext{
${ }^{12}$ For a further discussion of the criterion, as well as of alternative criteria of co-possibility, see Reference [10].
} 
in some other sense, or not at all events, or a causet (set of events) is not an event-like concept. The later witness intuition builds upon a concept of event in which the identity criteria for eventhood include having a fixed past. So in this sense of "events", an event cannot occur more than once and, moreover, in two senses: it cannot be repeated in either one history, or in multiple histories. If an event were multi-realizable by having parts (or instances) in alternative histories, one might explicate the notion of event's past in such a way that its past would comprise objects that are not co-possible. Such multi-realizable events have a room in analytic metaphysics. For the record, recall David Lewis's [8, p. 196] notion of non-fragile event, which serves as a basic block in his theory of causation. Lewis's non-fragile event is multi-realizable in alternative possible worlds, but (as I understand it) not in one possible world. So a non-fragile event might have occurred somewhat differently than it actually occurred. But it cannot re-occur after a time being. This suggests that perhaps causets are multi-realizable events.

Drawing on different senses of "events" might be confusing and so it would be better to use a different and more general term, "states". Clearly, the state of a given object can be repeated in the object's history and an object might hit upon the same state in its alternative histories. After all, we can perfectly imagine an object's state that can be reached along alternative histories of that object. To illustrate, a particular state of clutter on my desk may follow the Head-up result of a particular coin-flip, as well as the Tail-up result of that flip-after the coin has been removed from the desk. To extend this idea to states of an isolated system (or, of a world) requires states to not fully encode their pasts. Then the existence of a later state preceded by two other states does not means that these two latter states belong to the same history. These two states can be stages of two alternative processes that lead to the same state. As a result, with states the intuition of a later witness fades away.

Understanding causets as states removes the sting of Facts 8 and 11, at least to a certain extent. For any two states, they are followed, sooner or later, by another state. Furthermore, every typical state has more than one immediate predecessor (a parent state). This says more than that every typical state can be arrived along multiple paths. The last stages of these paths, before they converge on a state in question, are to be different. Let us take it tentatively for a sound albeit somewhat exuberant picture of emerging and criss-crossing possible evolutions.

There is an advantage of the turn to states: now the model of sequential growth exhibits features that are known from a typical way a physical theory is constructed. The sequential growth generates a state space (aka phase space). In the state space there are maximal sequences of states, one following another, by Fact $9 .{ }^{13}$ These sequences are discrete trajectories in the state space. Each non-maximal state belongs to multiple trajectories, which makes a dramatic contrast to never-crossing trajectories of the Hamiltonian formulation of classical mechanics. A typical state has many parents, each parent belonging to a different trajectory, and every non-maximal state has more than one child. Thus, multiple trajectories crisscross at each typical state (where "typical" means non-maximal and fulfilling the description given in footnote 11). This is a picture of exuberant possibilities that grow profusely, with almost any state being an

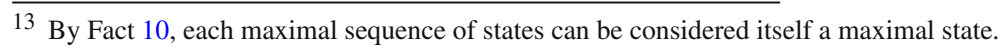


outgrowth of multiple alternative processes (encoded by trajectories). That opulence of possibilities might appear distressing but, no worries, there is way to tame it, namely, probabilities.

As a digression, we return to the later witness intuition: can we accommodate it in this framework? We noted that the intuition requires an event-concept with the identity criteria including sameness of the events' past. Clearly, a typical causet does not satisfy such criteria, as it can be produced along different causal paths. But can we still make good of this intuition in the causal sets programme? To this end we need to use a subtler notion of causet that encodes the causet's ancestry as well. A causet, understood as a state, typically has many ancestries, depending along which path it emerges. Thus, a simple way to encode a causet's ancestry is to associate it to a path in $C_{D}^{\text {fin }}$ leading from a singleton-based causet to the causet in question. As any two subsequent causets in this path are different by exactly one element, the result of this association is that the causet's elements are now numbered, with the numbering representing the order of creation leading to this causet. A multiple-realizable un-numbered causet is now differentiated into a family of numbered causets, each numbering representing a path leading to the causet's creation. For more info in numbered causets, see Appendix.

The set of numbered causets is strictly ordered by a relation based on an concept analogous to that of a link in $C_{D}^{f i n}$. It can be proven that no two numbered causets have an upper bound (understood as a numbered causet). Any two numbered causets always have a lower bound, however, and all paths through the forest of numbered causets begin with a singleton-based causet. In short, numbered causets form a branching tree. ${ }^{14} \mathrm{~A}$ branching tree format implies that two numbered causets have a common later witness iff they are members of a maximal chain of numbered causets. Since the right-hand side of this bi-conditional is (naturally) read as saying that the two causets are co-possible, the bi-conditional is just the statement of the later witness intuition.

By interpreting causets as repeatable states, we now read chains of causets producible in sequential growth as discrete criss-crossing trajectories in a state space. This somewhat unorderly picture is to be pruned by probabilistic constraints, to which we now turn.

\section{Probability to the Rescue?}

Although sequential growth produces an abundance of criss-crossing trajectories in the space of un-numbered causets, some trajectories might be barely probable, or improbable. By interpreting the assignment of zero probability to a trajectory as impossibility of this trajectory, one might obtain a less exuberant picture of the possibilities emerging from sequential growth. Introducing probabilities to the theory of causets involves two tasks. The first is purely formal, and address questions like what objects probabilities are defined on, and which constraints they are subject to. The other task is to show how numerical values of formally defined probabilities result from a physical process, as described by some equations. Both the tasks are addressed in Rideout [11] and Rideout and Sorkin [12]. In this commentary I focus on the formal task alone.

$\overline{14 \text { I owe this observations to D. Rideout, in a personal communication. }}$ 
To begin with, we should decide which framework, of un-numbered causets or of numbered causets, should be used for the introduction of probabilities. Although our exegesis of the above papers pushes us in opposite directions, we opt for un-numbered causets. ${ }^{15}$ The next decision is the following: to what objects are probabilities to be assigned?

\subsection{Transition Probabilities}

Probabilities are assigned to pairs $<$ parent, child $>$, written as $\mu(<$ parent, child $>)$, and interpreted as the probabilities of transitions from a parent to its child. The interpretation implies that the probability of the passage from a parent to its child is probabilistically independent from the parent's history. It follows that the probability of a passage from $B_{1}$ to $B_{2}$ along a given path $\gamma$ in $C_{D}^{f i n}$ is the product of transition probabilities of all parent-child links forming the path $\gamma$.

Next, the probabilities are subject to a "summing to unity" constraint.

\subsection{Summing to Unity}

Let $A$ be a causet with children $B_{1}, B_{2}, \ldots, B_{k}$. Then $\sum_{i=1}^{k} \mu\left(\left\langle A, B_{i}\right\rangle\right)=1$.

With this contraint, one can associate a Kolmogorovian probability space $\langle X, \mathcal{F}, p\rangle$ to each causet $A$. Here the base set $X$ is the set of all pairs $\left\langle A, B_{i}\right\rangle$, where $B_{1}, B_{2}, \ldots, B_{k}$ are the children of $A$ (or equivalently, $X$ is the set of all children of $A$ ). $\mathcal{F}$ is the field of subsets of $X$ and $p$ is the probability measure on $\mathcal{F}$, uniquely defined by transition probabilities $\mu$ on $X$. Clearly, $\mathcal{F}$ is finite, as any finite causet has finitely many children. ${ }^{16}$

The next condition is what Rideout and Sorkin [12] term the Bell causality condition. To state it, we need the concept of a precursor. The precursor of a link consists of exactly those elements of the link's parent that causally precede the new element in the link's child.

\subsection{Bell Causality Condition}

Let $C$ be an arbitrary causet based on $n$-elements proto-causets, $C_{1}, C_{2}$ be two children of $C$, whose new elements are $e_{1}$ and $e_{2}$, respectively. ${ }^{17}$ Consider then a "pruned" segment $B$ of $C$, which is the union of the preceqursor of link $\left\langle C, C_{1}\right\rangle$ and the prece-

15 The description of how a causet's children are to be counted for the summing to unity constraint requires numbered causets. But the idea that a causet can be reached along multiple paths, which underlies general covariance, is in line with un-numbered causets, but not with numbered causets.

16 Note that "summing to unity" delivers different numbers, depending on how a causet's children are counted. Typically a numbered causet has more children than its un-numbered cousin.

17 Since the set-theoretical representation of causets is somewhat multi-layered, giving a precise definition of concepts like precursor, or a child's new element requires lengthy and little-illuminating work. We thus leave the condition in a none too precise form, relying on the reader's ability to grasp it, and do the formal work, if needed. 
qursor of link $\left\langle C, C_{2}\right\rangle$. Construct then two children $B_{1}, B_{2}$ of $B$ by adding $e_{1}$ and $e_{2}$ to $B$, respectively. Then the condition requires the following:

$$
\frac{\mu\left(C, C_{1}\right)}{\mu\left(C, C_{2}\right)}=\frac{\mu\left(B, B_{1}\right)}{\mu\left(B, B_{2}\right)} .
$$

To comment on the condition, the "pruned" causet $B$ might contain substantially fewer elements than the initial causet $C$. Each element of $B$ causally precedes a new element in one child or the other. Thus, although the links $C, C_{1}$ and $C, C_{2}$ might be located somehere high in the tree produced by the sequential growth, the links $B, B_{1}$ and $B, B_{2}$ might be located significantly lower. The meaning of the equation is that as far as the quotient of transition probabilities with a fixed parent is concerned, the quotient does not depend on these elements of the parent that are causally unrelated to the children's new elements. From a more formal perspective, the condition imposes a contraint on how probability spaces associated with different causets are to be related.

The next condition intends to "gauge out" the paths along which a causet is produced. We left it to the end of this section, as it casts new light on the interpretation of causets.

\subsection{General Covariance}

For any $C \in \mathrm{C}_{\mathrm{D}}^{\text {fin }}$, if $\gamma$ and $\gamma^{\prime}$ are paths in $\mathrm{C}_{\mathrm{D}}^{\mathrm{fin}}$ that originate at the singleton-based causet and terminate at $C$, then the product of the transition probabilities along the links of $\gamma$ must be the same as the product of the transition probabilities along the links of $\gamma^{\prime}$.

Why should one identify the probabilities along various paths leading to a causet $C$ ? Each path encodes an order of becoming of $C$. Each path thus yields a different numbering of elements of $C$. Since by Definition 3, a new element cannot be placed below an old element, it is impossible that $x \prec y$, while $n(y) \leq n(x)$, where $n$ is a numbering function, and $\leq$ is the ordering of natural numbers. Accordingly, every path leading to $C$ induces an ordering $n$, called "natural", that is subject to this condition: if $x \prec y$, then $n(x)<n(y)$. (Note that this condition is a one-way implication rather than the equivalence. For a definition of numbering functions, incl. a natural numbering, see the Appendix). Thus, abstracting from numerical values, all natural numberings for a given causet $C$ agree on how causally related elements of $C$ are ordered. They disagree (still abstracting from particular numerical values) on causally unrelated elements, understood here as SLR elements. But this disagreement cannot be substantial. After all, for SLR events $\left\{e_{1}, e_{2}, \ldots, e_{n}\right\}$ the question of which was created first, does not admit a non-perspectival answer. If we ask at $e_{1}$, the answer is " $e_{1}$ ", if we ask at $e_{2}$, the answer is " $e_{2}$ ", etc. Rideout and Sorkin [12] thus concede that natural numberings are not meant "to carry any physical information". Since natural numberings for $C$ and paths leading to $C$ stand in one-to-one correspondence, the paths leading to $C$ do not carry any physical information either. This is the reason of why all paths leading to $C$ receive the same probability. But if the paths carry no physical information needed for diverging probability assignments, how can they, with 
the absence of differentiating physical information, be considered alternative possible paths to $C$ ?

General covariance casts a somewhat unfriendly light on our interpretation of elements of $\mathrm{C}_{\mathrm{D}}^{\mathrm{fin}}$ as states. Recall that a typical element $A \in \mathrm{C}_{\mathrm{D}}^{\mathrm{fin}}$ has multiple parents, and hence can be reached by multiple paths, all starting with the singleton-based causet. However, since the premises of General Covariance are satisfied, the probabilities of reaching $A$ along different paths should be the same. Now reading $A$ as a multi-realizable state, we get that no matter how this $A$ is arrived at, the probability of its production should be the same. This might appear somewhat strange, as we may imagine (drawing on our everyday's experience) that some paths leading to a given state are easier than the other. To explain away this weirdness, we turn to the argument for general covariance, only to learn that this argument concludes that paths are not physically real. But if they are not physically real, they can hardly play the role of alternative possible paths leading to $C$. After all, if the paths do not to carry any physical information, what information are they supposed to carry? The only answer, I think, is that this information regards our ways of representing reality rather than any information about reality itself, where ways of representing might result from the formal methods we use. Clearly, one needs a much more substantial differentiation of paths to consider them as alternative possible ways of producing a given causet.

Having seen the problems produced by the probabilistic constraint of general covariance, one might be tempted to reject it. Obviously, the constraint invokes the standard desideratum that physical laws be invariant under arbitrary differentiable coordinate transformations, with the underlying idea that a choice of coordinates is conventional, and hence should have any bearing on what the laws of nature look like. But, since causets do not live in differentiable manifolds and continuous spacetime is only apparent, there is no single clear-cut answer how to apply the idea underlying general covariance to causets. Probabilistic general covariance is just one proposal, and (we think) not a fortunate one. One rendering of the idea is to require that ordering of elements in a causet has no physical significance. This seems to imply that there is only physically meaningful way of becoming for any given causet. This is a rejection of indeterministic becoming.

\section{Discussion}

In this paper we have focused on the modal aspects of causets. Let us recall where the argumentation set out in this paper leads us. After proving a few facts about causets, we argued that causets cannot be understood as particular events, as objects that are not multi-realizable. For this concept of eventhood there is a persuasive criterion for co-possibility, which is based on the later witness intuition. Since any two causets in $C_{D}^{f i n}$ have an upper bound (i.e., a later witness, exactly), any two causets in $C_{D}^{\text {fin }}$ are co-possible, and hence $C_{D}^{\text {fin }}$ admits exactly one maximal set of co-possible causets, i.e., just one history, identified thus with $C_{D}^{f i n}$ itself. The modalities are trivialised and no room is left for substantial possibilities.

We were therefore compelled to turn to a different understanding of causets. As a typical causets has many parents and belongs to multiple maximal chains in $C_{D}^{\text {fin }}$, we 
Fig. 4 Two paths, i and ii, of the creation of causet $C$

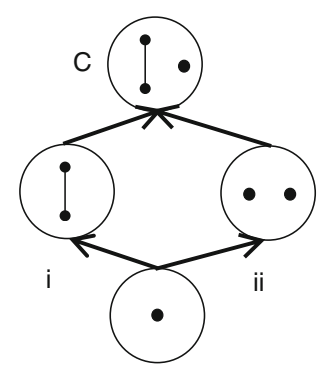

considered causets to be multi-realizable, like states or Lewisian non-fragile events. On this understanding, causets producible by sequential growth form a state space; maximal chains in this state space are (discrete) trajectories. The picture is in radical contrast to the orderly trajectories of classical physics, as all trajectories in $C_{D}^{\text {fin }}$ start at a (unique) minimal element and multiple trajectories cross at a typical state. Still we found the picture promising, as the theory is supposed to be indeterministic, and one might hope that probabilities will help to reduce the number trajectories. While examining the argument for general covariance, which is a constraint on the probabilities definable on causets, we found that by this argument, the multiplicity of paths leading to a given should not be considered physically real. The paths had better be taken as our different representations of one physical process. With this diagnosis we end up back in a familiar pickle: as now paths converging to a given causet $C$ are not thought of as alternative evolutions, whatever causets are below a given causet they are co-possible. By a familiar argument, drawing on Fact 8 , we get that $\mathrm{C}_{\mathrm{D}}^{\mathrm{fin}}$ is a (single) maximal set of co-possible causets, i.e. a (single) possible history. Is there a way out of this predicament, apart from rejecting substantial possibilities? We end this paper with two suggestions.

First, it seems to us that the appeal to the physical irrelevancy of the ordering of SLR events may take two forms, and these two forms are conflated in the causal sets theory. The first appeal is operative in the concept of un-numbered causet. Just by being un-numbered, the causet does not provide us with any information as to how a subset of its SLR elements is ordered (but it gives information on, e.g., how large is the subset). The second appeal is at work in the argument for general covariance, and it concerns paths of causets rather than a single causet. A (finite) path of causets represents a particular order of creation of a given causet. Figure 4 shows two paths leading to a simple causet $C$. Are these paths different? If one takes the order of creation as trumping relativity, the answer is affirmative: path (i) depicts a singletonbased causet giving birth to a causet with a single link, which in turn gives birth to a causet with one element causally un-related to a causally-related pair. Path (ii) tells a different story, about a singleton-based causet giving way to a causet based on a causally un-related pair, and finally - to a causet based on a triple, with one element un-related to a causally related pair. Note that since all the involved causets are unnumbered, there is no problematic ordering of causally unrelated elements in each causet. 
Things are different, however, if priority is given to relativity, to the point of ignoring the concept of creation. Then one is deemed to look at the final causet $C$, in a vain attempt to figure out an order of its elements. The attempt is vain, as it must involve the dubious task of assigning order of priority to SLR events.

Thus, to give a chance to alternative multiple possible histories in $\mathrm{C}_{\mathrm{D}}^{\mathrm{fin}}$, one option is to take the concept of the order of creation seriously: the advice is to let creation trump relativity. Since this move undermines the argument for General Covariance, for consistency's sake this constraint should not be postulated. This need not be done since the irrelevancy of the ordering of causally unrelated elements is already captured in un-numbered causets.

Our second suggestion of how to escape from the predicament is to re-think what it means, in terms of the causets theory, that two elements of a causet are not comparable by the causal ordering. In the present paper we followed the custom of causets theorists identifying causally incomparable elements with space-like related elements. However, in a fully fledged modal account, this identification simply appears to be wrong. Two events might be incomparable because they belong to different alternative evolutions, not because they belong to the same evolution and are space-like related. We leave, however, the task of developing this suggestion and examining its cogency to a future work.

\section{Appendix: On Numbered Causets}

In a numbered causet, its elements have partial identity as they are numbered. I call it "partial" because the identity of elements of the underlying set $D$ is forgotten. Still, by knowing a numbering, a given number can be associated to the appropriate elements of posets, whose base sets are subsets of $D$.

Formally, we define a (finite) numbered causet as a pair $\langle A, n\rangle$, where $A \in \mathrm{C}_{\mathrm{D}}^{\text {fin }}$ and $n$ is a numbering function for $A$, which we now explain.

Definition 12 (Numbering function for a causet)Let $A=\left\{\left\langle S_{\gamma}, \preceq_{\gamma}\right\rangle \mid \gamma \in \Gamma\right\} \in \mathrm{C}_{\mathrm{D}}^{\text {fin }}$ be based on $m$-elements proto-causets (with $\Gamma$ an appropriate index set). A function $n: \bigcup_{\gamma \in \Gamma} S_{\gamma} \mapsto\{1,2, \ldots, m\}$ is a numbering function for $A$ iff it satisfies the three conditions below:

(i) for each $\gamma \in \Gamma$, the restriction $n_{\mid S_{\gamma}}$ of $n$ to $S_{\gamma}$ is a bijection between $S_{\gamma}$ and $\{1,2, \ldots, m\}$

(ii) if $x \in S_{\gamma}$ and $y \in S_{\eta}(\gamma, \eta \in \Gamma)$ are equivalent (in the sense of being linked by an order-preserving isomorphism between $S_{\gamma}$ and $S_{\eta}$ ), then $n(x)=n(y)$, and

(iii) for every $\gamma \in \Gamma$, if $x \preceq_{\gamma} y$, then $n(x)<_{\mathbb{N}} n(y)$.

Denoting the set of finite numbered causets by ${ }_{n} C_{D}^{\text {fin }}$, analogously to Definition 3 we define a link in ${ }_{n} C_{D}^{f i n}$ and then, in terms of the link, the ordering $<$ of ${ }_{n} C_{D}$ fin ${ }^{18}$ It can be proved that $\left.{ }_{n} C_{D}^{\mathrm{fin}},<\right\rangle$ is a strict order and that any two elements of it have an

18 In contrast to a link in $C_{D}^{\text {fin }}$, a condition on numbering functions is needed, to the effect that numbering functions associated with a parent and a child, resp., agree on some sets. We leave the details to the reader. 
infimum (greatest lower bound) but no upper bound. Thus, $\left\langle{ }_{n} C_{D}^{f i n},<\right\rangle$ is a tree. We leave the proofs to the reader.

Open Access This article is distributed under the terms of the Creative Commons Attribution 4.0 International License (http://creativecommons.org/licenses/by/4.0/), which permits unrestricted use, distribution, and reproduction in any medium, provided you give appropriate credit to the original author(s) and the source, provide a link to the Creative Commons license, and indicate if changes were made.

\section{References}

1. Belnap, N.: Branching space-time. Synthese 92, 385-434. 'Postprint' archived at PhilSci Archive, (1992). http://philsci-archive.pitt.edu/archive/00001003

2. Bombelli, L., Lee, J., Meyer, D., Sorkin, R.D.: Space-time as a causal set. Phys. Rev. Lett. 59, 521-524 (1987)

3. Choquet-Bruhat, Y., Geroch, R.: Global aspects of the Cauchy problem in general relativity. Commun. Math. Phys. 14, 329-335 (1969)

4. Chruściel, P.T., Isenberg, J.: Nonisometric vacuuum extensions of vacuum maximal globally hyperbolic spacetimes. Phys. Rev. D 48(4), 16616-1628 (1991)

5. Dowker, F., Zalel, S.: Evolution of universes in causal set cosmology. Comptes Rendus Phys. 18, 246-253 (2017)

6. James, W.: The dilemma of determinism. Unit. Rev. 22, 193-224 (1884)

7. Lewis, D.: New work for a theory of universals. Australas. J. Philos. 61, 343-377 (1983)

8. Lewis, D.: Postscripts to Causation. In: Philosophical Papers Volume II, pp. 172-213. Oxford University Press, Oxford (1986)

9. Malament, D.B.: The class of continuous timelike curves determines the topology of spacetime. J. Math. Phys. 18, 1399-1404 (1977)

10. Müller, T.: Alternatives to histories? Employing a local notion of modal consistency in branching theories. Erkenntnis 79(3), 343-364 (2014)

11. Rideout, D. P.: Dynamics of Causal Sets. PhD thesis, Graduate School of Syracuse University (2001)

12. Rideout, D.P., Sorkin, R.D.: A classical sequential growth dynamics for causal sets. Phys. Rev. D 61, 024002 (2000)

13. Sorkin, R.: Logic is to the quantum as geometry is to gravity. In: Murugan, J., Weltman, A., Ellis, G.F.R. (eds.) Foundations of Space and Time: Reflections on Quantum Gravity, pp. 363-384. Cambridge University Press, Cambridge (2012)

14. Wüthrich, C.: The structure of causal sets. J. Gen. Philos. Sci. 43, 223-241 (2012)

15. Wüthrich, C., Callender, C.: What becomes of a causal set? Br. J. Philos. Sci. 68(3), 907-925 (2017)

16. Wüthrich, C.: Spacetime from causality: causal set theory. In: Huggett, N., Wüthrich, C. (eds.) The Emergence of Spacetime in Quantum Theories of Gravity. Oxford University Press, Oxford (2019)

Publisher's Note Springer Nature remains neutral with regard to jurisdictional claims in published maps and institutional affiliations. 